University of Wollongong

Research Online

Faculty of Engineering - Papers (Archive)

Faculty of Engineering and Information

Sciences

2012

\title{
Biomorphic porous graphitic carbon for electromagnetic interference shielding
}

Qinglei Liu

Shanghai Jiao Tong University

Jiajun Gu

Shanghai Jiao Tong University

Wang Zhang

Shanghai Jiao Tong University

Yoshinari Miyamoto

Osaka University

Zhixin Chen

University of Wollongong, zchen@uow.edu.au

See next page for additional authors

Follow this and additional works at: https://ro.uow.edu.au/engpapers

Part of the Engineering Commons

https://ro.uow.edu.au/engpapers/5051

\section{Recommended Citation}

Liu, Qinglei; Gu, Jiajun; Zhang, Wang; Miyamoto, Yoshinari; Chen, Zhixin; and Zhang, Di: Biomorphic porous graphitic carbon for electromagnetic interference shielding 2012, 21183-21188.

https://ro.uow.edu.au/engpapers/5051

Research Online is the open access institutional repository for the University of Wollongong. For further information contact the UOW Library: research-pubs@uow.edu.au 


\section{Authors}

Qinglei Liu, Jiajun Gu, Wang Zhang, Yoshinari Miyamoto, Zhixin Chen, and Di Zhang 


\title{
Materials Chemistry
}

Cite this: J. Mater. Chem., 2012, 22, 21183

wWw.rsc.org/materials

PAPER

\section{Biomorphic porous graphitic carbon for electromagnetic interference shielding}

\author{
Qinglei Liu, ${ }^{a}$ Jiajun Gu, ${ }^{a}$ Wang Zhang, ${ }^{a}$ Yoshinari Miyamoto, ${ }^{b}$ Zhixin Chen ${ }^{c}$ and Di Zhang ${ }^{* a}$
}

Received 13th July 2012, Accepted 24th August 2012

DOI: $10.1039 / \mathrm{c} 2 \mathrm{jm} 34590 \mathrm{k}$

\begin{abstract}
Using a kind of plant biomass (coconut shell) as template and carbon precursor, we prepare biomorphic porous carbon materials for electromagnetic interference shielding application. The carbon frameworks of the porous carbon materials are modified by in situ formation of graphitic nanostructures in a catalytic graphitization process, leading to well-tailored electrical conductivity of the resultant materials. The modified porous carbon materials exhibit shielding effectiveness of $40 \mathrm{~dB}$ over the X-band frequency, and the shielding by absorption is as high as $91 \%$, indicating an absorptiondominant shielding mechanism. The high absorption contribution is attributed to the coupled effects of the biomorphic porous structures and graphitic nanostructures of the porous graphitic carbon.
\end{abstract}

\section{Introduction}

Electromagnetic interference (EMI) shielding both at radio and microwave frequencies is still in great demand for communication technology. ${ }^{1-4}$ In order to meet a variety of EMI shielding requirements, much attention has been paid to the development of advanced EMI shielding materials. Recently, conductive polymer composites have attracted much attention for use as EMI shielding materials due to their advantages, such as lightweight, high versatility, and easy processablity. ${ }^{5-7}$ The electrical conductivity and shielding performance of these polymer composites are closely related to intrinsic conductivity, aspect ratio, content, and dispersion of the fillers. As another promising shielding candidate, carbon foam, a kind of porous carbon material, has also received a great deal of attention. ${ }^{8-10}$ In addition to being lightweight, carbon foam is electrically conductive, and thermally and chemically stable. Hence, it has promising shielding applications in harsh environments including aircraft, spacecraft, shelters, and other electronic enclosures. Carbon foam materials have exhibited excellent EMI shielding as well as microwave absorption capacity, ${ }^{11}$ which could be closely ascribed to their three-dimensionally (3D) interconnected carbon framework. ${ }^{12}$ To date, however, few works have been carried out to explore the effect of the 3D carbon framework modification on the EMI shielding. In addition, the shielding mechanisms still remain unclear for these porous carbon materials.

Porous carbon materials with 3D interconnected framework are generally prepared by comparatively complicated foaming or template-processing using various polymers as carbon

${ }^{a}$ State Key Laboratory of Metal Matrix Composites, Shanghai Jiao Tong University, Shanghai 200030,P. R. China.E-mail: zhangdi@sjtu.edu.cn; Fax: +862134202749

${ }^{b}$ Joining and Welding Research Institute, Osaka University, Japan cFaculty of Engineering, University of Wollongong, Wollongong, NSW2522, Australia precursors. ${ }^{13-15}$ Until now, little effort has been paid to the development of these functional porous carbons by utilizing plant biomass. Plant biomass is frequently a highly $3 \mathrm{D}$ interconnected porous material with carbon, hydrogen, and oxygen as major chemical constitutive elements. One possible reason for the less attention paid to plant biomass may be partly related to its complex hierarchical and anisotropic structures, which are extremely difficult to synthetically replicate. In addition, porous carbon materials derived from the plant biomass are mainly composed of non-graphitizing carbon. This kind of carbon texture is quite resistant to graphitization under a direct graphitization process, ${ }^{16}$ which may hinder the development of functional carbon materials from the plant biomass. Since plant biomass is considered an ideal renewable resource due to its lowcost and abundance (about 180 billion tons produced annually), ${ }^{17}$ here we report a general and facile method to fabricate biomorphic porous carbon (BPC) with a quasi-foam structure for effective EMI shielding application by using plant biomass as the raw material. Coconut shell, a common kind of agricultural residue used for the production of activated carbon, was chosen as the biotemplate and carbon precursor in this work to produce BPC with 3D interconnected framework. Considering the important role of electrical conductivity for EMI shielding, we made a carbon framework modification to increase the graphitization degree of the BPC by a catalytic graphitization process below $1000{ }^{\circ} \mathrm{C}$. The catalytic graphitization made it possible to produce graphitic nanostructures under mild conditions by using transition metals, ${ }^{18-20}$ thereby broadening applicability of this approach in the exploration of functional carbons from biomass. In order to clarify the EMI shielding mechanisms of the porous carbon-based materials, we also investigated the contributions of the reflection and absorption in the overall EMI shielding effectiveness (SE). In addition, effects of porous structures and electrical conductivity on the EMI shielding behaviors of the obtained materials were discussed herein as well. 


\section{Experimental}

\subsection{Preparation of BPCs}

Coconut shell was crushed and ground into powders with a blender. The powders were directly carbonized at $500-1000^{\circ} \mathrm{C}$ in vacuum to produce BPCs. In order to produce the BPCs with graphitic frameworks, the coconut shell powders were impregnated in an iron nitrate solution by a wet-impregnation method before a carbonization sintering process. In a typical process, the weight ratio of Fe ion to the coconut shell was $1: 49$. The carbonization process in vacuum was described as follows: the samples were heated from ambient temperature to $450{ }^{\circ} \mathrm{C}$ at a heating rate of $5^{\circ} \mathrm{C} \mathrm{min}^{-1}$, and then to $1000{ }^{\circ} \mathrm{C}$ at a heating rate of $1{ }^{\circ} \mathrm{C} \mathrm{min}^{-1}$. The samples were held at $1000^{\circ} \mathrm{C}$ for an hour and then cooled to room temperature. For simplicity, the produced $\mathrm{Fe}$-containing porous carbons with graphitic frameworks were denoted as BPC-Gs. Acid treatment was used to remove Fe in the BPC-Gs, and the obtained samples were denoted as BPC- $\mathrm{G}_{\mathrm{R}}$.

\subsection{Characterization}

As-prepared samples were characterized with X-ray diffraction (XRD) using a Rigaku D/max 2550VL/PC system operated at 40 $\mathrm{kV}$ and $40 \mathrm{~mA}$ with $\mathrm{Cu} \mathrm{K} \alpha$ radiation, at a scan rate of $5^{\circ} \mathrm{min}^{-1}$ and a step size of 0.050 in $2 \theta$. The microstructures of the BPCs were studied using a FEI (Sirion200) scanning electron microscope (SEM). The graphitic microstructure of the BPC-Gs was investigated using a JEOL JEM-2010 transmission electron microscope (TEM) operated at $200 \mathrm{kV}$.

In order to measure electrical conductivity and EMI shielding effectiveness of the BPCs with and without graphitic frameworks, the prepared powders were mixed well with $30 \mathrm{wt} \%$ phenol formaldehyde (PF) resin (BUEHLER: PhenoCure 640). The mixture was then hot-pressed into sheet samples of $4 \mathrm{~mm}$ in thickness. Electrical conductivity of the BPC, BPC-G, and BPC$\mathrm{G}_{\mathrm{R}}$ sheets was measured via a four-probe method (Keithley 195A digital multimeter). The current contacts at both ends of a rectangular sheet were coated with silver paint to reduce the contact resistance. For the samples beyond the measurement range, a digital super megohmmeter (TOADKK DSM-8103) was used to measure their electrical conductivities. EMI shielding effectiveness (SE) over the X-band was measured using a microwave vector network analyzer (HP-E 8364B) with a rectangular waveguide setup. The samples were cut precisely into $22.86 \mathrm{~mm} \times$ $10.16 \mathrm{~mm}$ to fit the waveguide sample holder. A total of 201 data points for the scattering parameters (reflection and transmission) were taken by the network analyzer over the measured frequency range. The EMI SE of a material was defined as the ratio of transmitted power to incident power and could be represented by $\mathrm{SE}=10 \log \left(P_{\mathrm{I}} / P_{\mathrm{T}}\right)$, where $P_{\mathrm{I}}$ and $P_{\mathrm{T}}$ were the power incident on and transmitted through a shielding material.

\section{Results and discussion}

\subsection{Microstructures of BPC with and without graphitic frameworks}

Porous carbon produced from plant biomass by a direct carbonization process has a biomorphic structure that is inherited from the carbon precursor. As shown in Fig. 1a, BPC derived from coconut shell had a quasi-foam structure consisting of numerous cells, with a cell diameter of around $10 \mu \mathrm{m}$. The BPC showed a 3D interconnected carbon framework with a hierarchical porous structure (Fig. 1b). Different from carbon foam, ${ }^{13}$ the BPC exhibited an obviously anisotropic structure. Since the morphology of the BPC-G is similar to that of the BPC, SEM images of BPC-G are not shown here. The carbon framework, as indicated in the XRD results (Fig. 1i), was mainly composed of amorphous carbon even under carbonization temperatures as high as $1000{ }^{\circ} \mathrm{C}$, owing to its intrinsic feature of non-graphitizing carbon.

Graphitic nanostructures can be obtained from the nongraphitizing carbon under a relatively low temperature $(<1000$ ${ }^{\circ} \mathrm{C}$ ) by means of heterogeneous graphitization carried out with the aid of catalysts. After impregnation in the iron precursor, the coconut shell reacted with the iron precursor to produce the nano-sized iron particles during the heating process in vacuum (Fig. 1c). The produced iron nanoparticles were catalytically active to surrounding disordered carbon. As a result, the growth of graphitic nanostructures occurred above $700{ }^{\circ} \mathrm{C}$ (Fig. 1d), leading to an improvement in graphitization degree of the resultant carbon materials. As the temperature increased, the intensity of the diffraction peak in the XRD pattern ascribed to the graphitic carbon became stronger as showed in Fig. 1i, indicating an increase in the graphitization degree with temperature (Fig. 1e). At a temperature of $1000{ }^{\circ} \mathrm{C}$, locally interconnected graphitic nanostructures, as shown in Fig. 1f-h, were obtained as a result of sufficient catalytic graphitization of the iron nanoparticles to the surrounding disordered carbon. The dissolution-precipitation mechanism was adopted to explain this solid catalytic graphitization process, where graphitic nanostructures were formed through a process involving the dissolution of amorphous carbon into catalysts followed by the precipitation of graphitic carbons. ${ }^{18}$ It was reported that the graphitic nanostructures produced by the catalytic graphitization, such as core-shell, ribbon-like, spiral and irregular graphitic structures, were associated with carbon precursors as well as processing. ${ }^{20}$ Although the obtained graphitic nanostructures are in different forms, they should play the same role in the enhancement of the electrical conductivity of the resulting BPC-Gs.

\subsection{Electrical conductivity and EMI shielding}

Electrical conductivities of carbon materials are closely dependent on the graphitization degree. Electrical conductivity of carbon foam was observed to be distinctly improved as the carbonization temperature increased from 700 to $760{ }^{\circ} \mathrm{C} .{ }^{12}$ Fig. 2a illustrates the electrical conductivity of BPCs, BPC-Gs, and $B P C-G_{R}$ as a function of heat-treatment temperature. For the BPCs, the electrical conductivity increased by five orders of magnitude as the temperature increased from 500 to $1000^{\circ} \mathrm{C}$. In comparison, a much sharper increase in electrical conductivity of the BPC-Gs was obtained owing to the formation of the graphitic nanostructures on the $3 \mathrm{D}$ porous carbon framework. At $1000{ }^{\circ} \mathrm{C}$, for instance, the conductivity of the BPC-G was $7.4 \times 10^{2} \mathrm{~S} \mathrm{~m}^{-1}$, nearly five orders of magnitude greater than that of the BPC, 


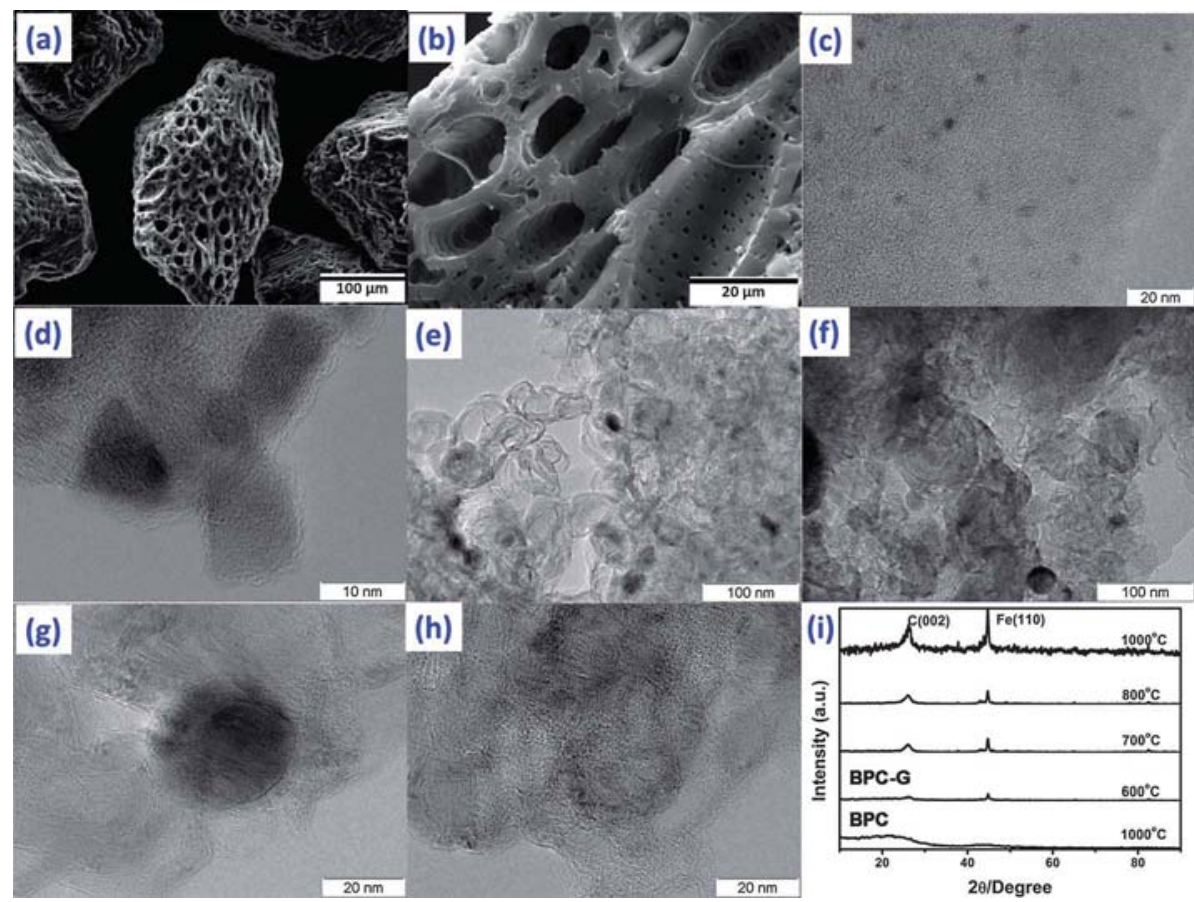

Fig. 1 (a and b) SEM images of BPC, (c-f) TEM images of BPC-Gs obtained at 600, 700, 800 and $1000{ }^{\circ} \mathrm{C}$, respectively, (g and h) magnified TEM images of the graphitic nanostructure catalytically formed at $1000^{\circ} \mathrm{C}$, and (i) XRD patterns of the $\mathrm{BPC}\left(1000^{\circ} \mathrm{C}\right)$ and $\mathrm{BPC}-\mathrm{Gs}\left(600-1000{ }^{\circ} \mathrm{C}\right)$.
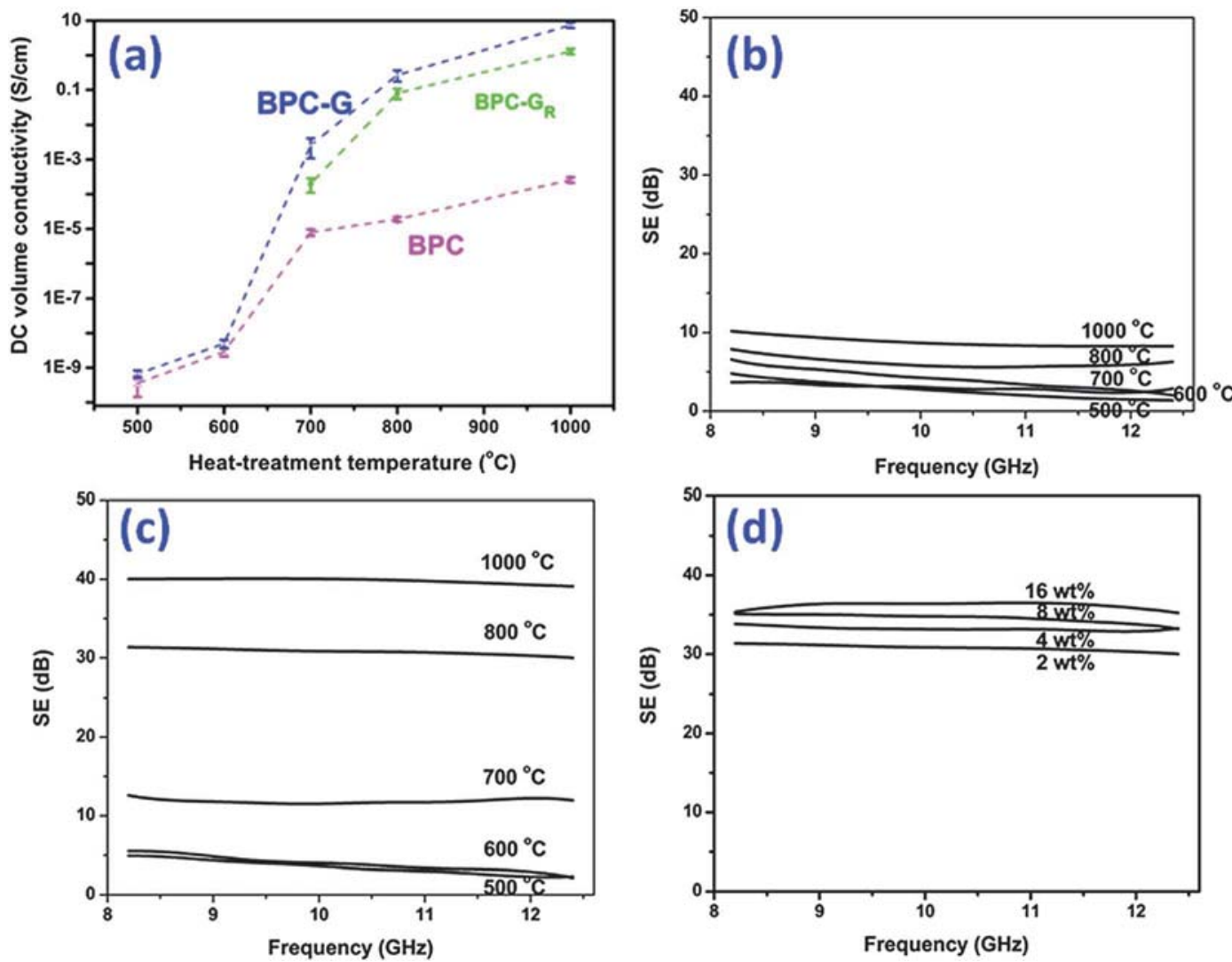

Fig. 2 (a) Electrical conductivity of BPCs, BPC-Gs and BPC- $G_{R}$ (after the removal of Fe) as a function of heat-treatment temperature, shielding effectiveness of BPCs (b) and BPC-Gs (c) as a function of heat-treatment temperature over X-band frequency, and (d) shielding effectiveness of BPC-Gs derived at $800{ }^{\circ} \mathrm{C}$ as a function of Fe content over X-band frequency. 

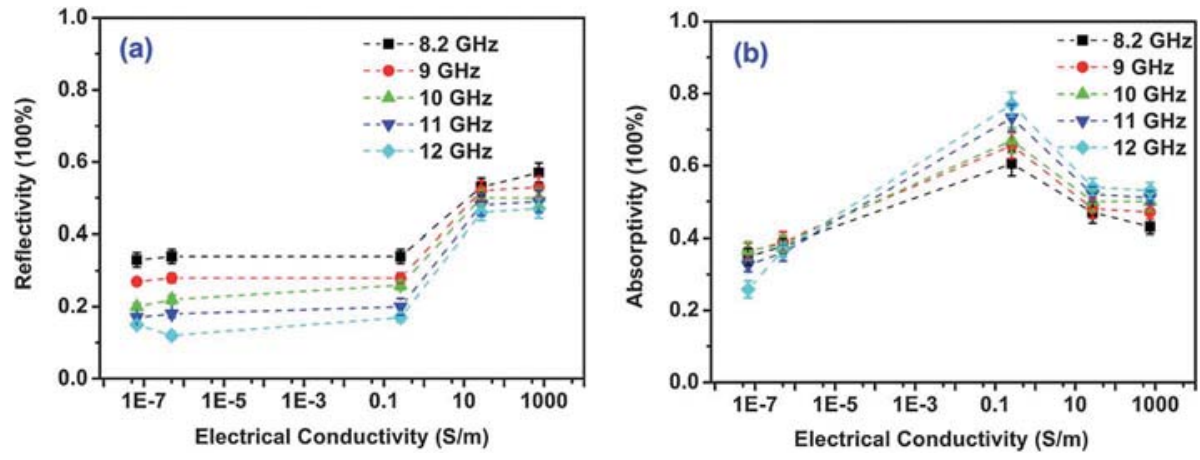

Fig. 3 (a) Reflectivity and (b) absorptivity for BPC-Gs as a function of electrical conductivity.

Table 1 Contributions of reflection and absorption in the overall EMI $\mathrm{SE}$ at $8.2 \mathrm{GHz}$ of BPC-Gs as a function of electrical conductivity

\begin{tabular}{lrrrl}
\hline $\begin{array}{l}\text { Electrical conductivity } \\
\left(\mathrm{S} \mathrm{m}^{-1}\right)\end{array}$ & $\mathrm{SE}(\mathrm{dB})$ & $\mathrm{SE}_{R}(\mathrm{~dB})$ & $\mathrm{SE}_{A}(\mathrm{~dB})$ & $\begin{array}{l}\% \text { SE by } \\
\text { absorption }\end{array}$ \\
\hline $6.7 \times 10^{-8}$ & $4.9 \pm 0.1$ & $1.7 \pm 0.1$ & $3.2 \pm 0.1$ & 65 \\
$5.0 \times 10^{-7}$ & $5.5 \pm 0.2$ & $1.8 \pm 0.1$ & $3.7 \pm 0.1$ & 67 \\
$2.6 \times 10^{-1}$ & $12.6 \pm 0.1$ & $1.8 \pm 0.1$ & $10.8 \pm 0.1$ & 86 \\
$2.7 \times 10^{1}$ & $31.3 \pm 0.3$ & $3.3 \pm 0.2$ & $28.1 \pm 0.2$ & 90 \\
$7.4 \times 10^{2}$ & $40.0 \pm 0.2$ & $3.7 \pm 0.1$ & $36.4 \pm 0.1$ & 91 \\
\hline
\end{tabular}

which was mainly ascribed to the formation of locally interconnected graphitic nanostructures as shown in Fig. 2a.

Fig. $2 \mathrm{~b}$ and $\mathrm{c}$ shows the EMI SE of BPCs and BPC-Gs as a function of carbonization temperature over X-band frequency. It was observed that the SE was almost independent of frequency across the measured region, but highly dependent on the carbonization temperature. The highest SE of the BPC at 1000 ${ }^{\circ} \mathrm{C}$ was below $10 \mathrm{~dB}$ due to its low electrical conductivity (Fig. 2b). In contrast, the BPC-Gs showed higher SE values at the same temperature since they had larger electrical conductivity. The SE value of the BPC-G at $1000^{\circ} \mathrm{C}$ was around $40 \mathrm{~dB}$ (Fig. 2c) over the whole $\mathrm{X}$-band, indicating an excellent shielding ability of these biomorphic quasi-foam carbon materials. Fig. 2d compares the SE of BPC-Gs containing different Fe contents derived at $800^{\circ} \mathrm{C}$. The results show that only $5 \mathrm{~dB}$ additional SE was obtained as the $\mathrm{Fe}$ content increased from 2 to $16 \mathrm{wt} \%$, indicating that a small amount of Fe addition was enough for obtaining desired shielding performance by creating graphitic nanostructures on the $3 \mathrm{D}$ porous carbon framework.

\subsection{EMI shielding mechanisms}

3.3.1 Contributions of reflection and absorption. It is known that the mechanisms of EMI shielding can be attributed to reflection, absorption, and multiple reflections. The shielding mechanisms of carbon foam, to our knowledge, have not been well reported. In order to investigate the effect of such a macroporous structure with 3D carbon framework on EMI shielding, reflectivity and absorptivity of the BPC-Gs $(2 \mathrm{wt} \% \mathrm{Fe})$ toward an incident electromagnetic power were obtained as a function of electrical conductivity, as shown in Fig. 3, by calculating the measured data of the transmitted power $(T)$ and reflected power $(R)$. The absorbed power $(A)$ can be calculated by the equation $A=I-(T+R)$, where $I$ is the applied incident power. It should be noted that the measured $R$ includes not only the power that has been reflected from the external surface, but

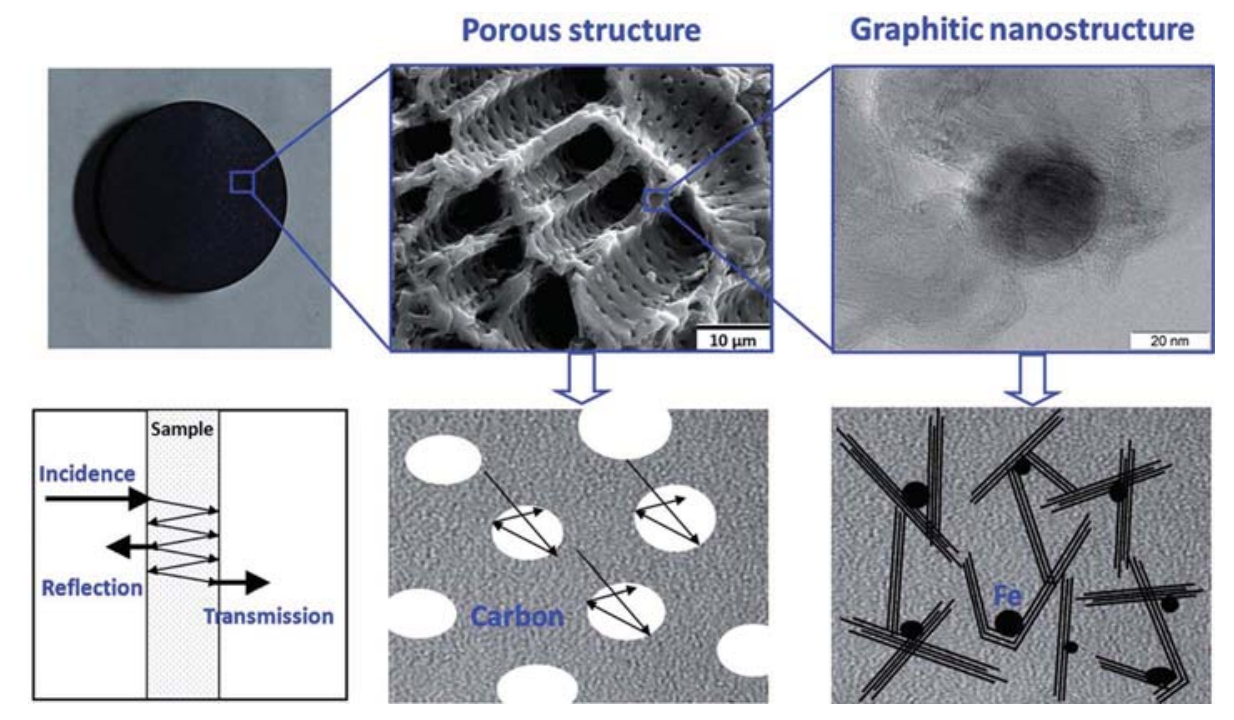

Fig. 4 Schematic illustration of shielding mechanisms for BPC-Gs. 
also contributions of internal surface reflection and multiple reflections as well. ${ }^{10}$

It can be seen from Fig. 3 that the reflectivity of the BPC-G sheets increased with electrical conductivity; whereas the absorptivity first increased with electrical conductivity then decreased again. Such a decrease in absorptivity could be attributed to the lower power transmitted into the sample as a result of the better reflection at a high conductivity. It is interesting that the amount of energy blocked by absorption was higher than that blocked by reflection for the majority of the measured electrical conductivities, and this trend was much more apparent at higher frequencies. This phenomenon is quite different from that of conductive polymer composites, such as single-walled carbon nanotube/polymer and multi-walled carbon nanotube/polymer composites. For these solid conductive polymer composites with similar electrical conductivities, reflectivity is much larger than absorptivity over the X-band frequency. ${ }^{5,7}$ Although the overall SE of a shielding material increases as the conductivity increases, the scientific relation between the EMI SE and electrical conductivity has not been clarified yet. ${ }^{1,7}$ As for the conductive BPC-G sheets, it seems that there was a threshold value in electrical conductivity, beyond which the BPC-G sheets began to have a drastic increase in reflectivity toward the incident microwave power as illustrated in Fig. 3a. The threshold value was slightly associated with the processing parameters (e.g., thickness of the sheets), but was almost in the range of $10^{-1}$ to 10 $\mathrm{S} \mathrm{m}{ }^{-1}$. As the conductivity of BPC-G sheet was below the threshold value, the reflectivity toward the incident electromagnetic power showed little improvement with the increased conductivity (Fig. 3a).

It can also be seen that the reflectivity at low frequency was larger than that at high frequency. For example, the BPC-G sheet with $7.4 \times 10^{2} \mathrm{~S} \mathrm{~m}^{-1}$ conductivity had a 0.57 reflectivity at 8.2 $\mathrm{GHz}$ but a 0.47 reflectivity at $12 \mathrm{GHz}$. This result is in accordance with previously reported shielding performance of the conductive polymeric composites at X-band frequency. ${ }^{5}$

Reflectivity and absorptivity are quantitative characteristics of power balance as electromagnetic radiation is incident on a shield. In order to evaluate the contributions of reflection and absorption to the overall shielding, SE values of the BPC-Gs were resolved into absorption loss $\left(\mathrm{SE}_{A}\right)$ and reflection loss $\left(\mathrm{SE}_{R}\right)$ using the power balance data, which could be described as

$$
\begin{aligned}
& \mathrm{SE}_{R}=10 \log \frac{I}{I-R} \\
& \mathrm{SE}_{A}=10 \log \frac{I-R}{T}
\end{aligned}
$$

$$
\begin{aligned}
\text { Overall } \mathrm{SE} & =\mathrm{SE}_{R}+\mathrm{SE}_{A}=10 \log \frac{I}{I-R}+10 \log \frac{I-R}{T} \\
& =10 \log \frac{I}{T}
\end{aligned}
$$

According to the above equations in the case of total reflection, values of the $\mathrm{SE}$ and $\mathrm{SE}_{R}$ are infinite. In the case of nonreflection, the $\mathrm{SE}_{R}$ value is zero. This indicates that the overall shielding is attributed to the absorption loss, though these $\mathrm{SE}_{R}$ and $\mathrm{SE}_{A}$ values involve contributions of multiple reflections and interference of waves. In addition, it should be noted that absorptivity is a value describing the ratio of power dissipated by the sample toward the overall incident power, while $\mathrm{SE}_{A}$ is a measure of the ability to attenuate the electromagnetic power that has transmitted into the sample. Table 1 lists the contributions of reflection and absorption in the overall EMI SE at 8.2 $\mathrm{GHz}$ of the BPC-Gs as a function of electrical conductivity. It can be observed that regardless of the electrical conductivity, SE contributed by absorption was higher than that by reflection, indicating an absorption-dominant shielding mechanism of the BPC-G materials. In addition, the EMI SE by absorption increased with the conductivity over the measured range. For example, for the sample with $7.4 \times 10^{2} \mathrm{~S} \mathrm{~m}^{-1}$ conductivity, the SE by absorption increased up to $91 \%$, demonstrating the excellent absorption ability of the BPC-G sheet toward the incident radiation in the $\mathrm{X}$-band frequency.

3.3.2 Effects of pore structures. As proved above, the BPC-G sheets have excellent absorption toward the incident microwave at $8.2-12.4 \mathrm{GHz}$. This result could be closely associated with the graphitic frameworks as well as the pore structures of the materials. A carbon-based shielding material must have mobile charge carriers (electrons or holes) to have a good SE toward the electromagnetic field. In this case, electrical conductivity, a characteristic of the number of mobile charge carriers, can be controlled over a large range as shown in Fig. 2. For a biomorphic porous structure with low conductivity, the SE value is very low, indicating a limited role played by the porous structures with low conductivity. As the conductivity increases, the pore structures should play more important roles in the shielding performance of the carbon materials. First, the pore structures would decrease the permittivity of materials. A porous material can be treated as an effective medium as a mixture of its components and air. The effective permittivity of a porous material can be determined by the Maxwell-Garnett (MG) theory. ${ }^{12}$ As a result, porous structures decrease the effective permittivity, and make a positive contribution to impedance match, indicating a lower reflection of the electromagnetic field achieved by the porous structures than by solid structures. Second, the pore structures would contribute to the $\mathrm{SE}_{A}$ by increasing the reflection and multiple reflections inside the porous carbon materials. Although it requires more study to demonstrate the contribution of the multiple reflections to the overall SE, multiple reflections should cause more energy to be dissipated inside because they make the microwave travel a longer distance in the carbon materials. To summarize, the pore structures play a positive role in increasing absorption contribution to the overall SE, but more investigations are still required to clarify the effect of the pore structures on the overall SE.

Based on the analyses above, a schematic diagram is proposed to describe shielding mechanisms of the BPC-Gs, as illustrated in Fig. 4. The BPC-Gs have a hierarchical quasi-foam structure originated from natural precursor. The electrical conductivity of the porous structure could be well-tuned over a large range by modifying the interconnected carbon framework. In passing through a BPC-G shield, an applied electromagnetic wave would be attenuated by reflection, absorption, and multi-reflections. The energy ratio attenuated by each portion is closely associated with the porous structure, electrical conductivity, and geometry of the shield. The porous structures have few shielding effects on 
the electromagnetic wave at low conductivities, but would make an important contribution to absorption loss at high conductivity by increasing the reflection and multiple reflections of the power transmitted into the sample. The graphitic nanostructures formed on the 3D carbon framework provide mobile charge carriers and conductive channels as the sheet is subjected to an electromagnetic field. This means that the porous structures and graphitic nanostructures have a combined effect on the increment of the absorption loss and overall SE. More investigations are required to clarify the scientific relationship between the parameters of pores, such as the pore size and porosity, the electrical conductivity and EMI SE by reflection and absorption.

\section{Conclusions}

Biomorphic porous carbon structures have been prepared by utilizing natural materials as templates and carbon precursors for EMI shielding. Electrical conductivity of the BPC-Gs is well tuned over a large range by creating graphitic nanostructures on the carbon framework. The BPC-G can have a SE of $40 \mathrm{~dB}$ over the $\mathrm{X}$-band frequency and the SE by absorption is as high as $91 \%$, indicating an absorption-dominant shielding mechanism of these porous carbon materials. This high absorption contribution is attributed to the combined effects of the porous structures and graphitic nanostructures of the BPC-G samples. Porous structures present a positive contribution to the absorption loss as the BPC-G samples have high conductivity. This study would contribute to the design and fabrication of porous structures for cost-efficient, light-weight, and effective EMI shielding and microwave absorption materials.

\section{Acknowledgements}

This work was supported by the National Natural Science Foundation of China (no. 51001070, 51202145), Shanghai
Science and Technology Committee (no. 10JC1407600), 973

National Project (no. 2011CB922202), Research Fund for the Doctoral Program of Higher Education of China. We also thank SJTU Instrument Analysis Center for the measurements.

\section{References}

1 D. D. L. Chung, Carbon, 2001, 39, 279.

2 M. Mahmoodi, M. Arjmand, U. Sundararaj and S. Park, Carbon, 2012, 50, 1455

3 G. A. Gelves, M. H. Al-Saleh and U. Sundararaj, J. Mater. Chem., 2011, 21, 829 .

4 M. Arjmand, M. Mahmoodi, G. A. Gelves, S. Park and U. Sundararaj, Carbon, 2011, 49, 3430.

5 Z. Liu, G. Bai, Y. Huang, Y. Ma, F. Du, F. Li, T. Guo and Y. Chen, Carbon, 2007, 45, 821.

6 A. Gupta and V. Choudhary, Compos. Sci. Technol., 2011, 71, 1563.

7 M. H. Al-Saleh and U. Sundararaj, Carbon, 2009, 47, 1738.

8 Y. Yonglai, M. C. Gupta, K. L. Dudley and R. W. Lawrence, Nano Lett., 2005, 5, 2131.

9 A. Fletcher, M. C. Gupta, K. L. Dudley and E. Vedeler, Compos. Sci. Technol., 2010, 70, 953 .

10 J. M. Thomassin, C. Pagnoulle, L. Bednarz, I. Huynen, R. Jerome and C. Detrembleur, J. Mater. Chem., 2008, 18, 792.

11 F. Moglie, D. Micheli, S. Laurenzi, M. Marchetti and V. M. Primiani, Carbon, 2012, 50, 1972.

12 Z. Fang, C. Li, H. Sun, H. Zhang and J. Zhang, Carbon, 2007, 45, 2873.

13 C. Chen, E. B. Kennel, A. H. Stiller, P. G. Stansberry and J. W. Zondlo, Carbon, 2006, 44, 1535.

14 J. Lee, J. Kim and T. Hyeon, Adv. Mater., 2006, 18, 2073.

15 A. B. Fuertes and T. A. Centeno, J. Mater. Chem., 2005, 15, 1079 .

16 D. W. McKee, Annu. Rev. Mater. Sci., 1973, 3, 195.

17 P. Maki-Arvela, T. Salmi, B. Holmbom, S. Willfor and D. Y. Murzin, Chem. Rev., 2011, 111, 5638.

18 M. Sevilla and A. B. Fuertes, Chem. Phys. Lett., 2010, 490, 63.

19 F. J. Maldonado-Hodar, C. Moreno-Castilla, J. Rivera-Utrilla, Y. Hanzawa and Y. Yamada, Langmuir, 2000, 16, 4367.

20 M. Sevilla and A. B. Fuertes, Carbon, 2006, 44, 468. 